PHYSICAL REVIEW D 95, 069908(E) (2017)

\title{
Erratum: Supersymmetric electric-magnetic duality of hypergravity \\ [Phys. Rev. D 90, 045029 (2014)]
}

Claudio Bunster, Marc Henneaux, Sergio Hörtner, and Amaury Leonard

(Received 23 March 2017; published 31 March 2017)

DOI: $10.1103 /$ PhysRevD.95.069908

There is an incorrect matrix $\gamma^{0}$ multiplying the right-hand side of Eq. (3.15). The corrected Eq. (3.15) should read

$$
\begin{aligned}
\mathcal{F}_{k}= & i\left[\partial_{k} \Xi-2 \partial^{l} \psi_{k l}+\partial_{k} \psi\right. \\
& +\gamma_{k} \gamma^{l} \partial_{l} \Xi+2 \gamma^{l} \gamma^{m} \partial_{l} \psi_{k m} \\
& \left.-\gamma_{k} \gamma^{l} \partial_{l} \psi+2 \gamma_{k} \gamma^{l} \partial^{m} \psi_{l m}\right] .
\end{aligned}
$$

This misprint has no incidence on the analysis of the paper and on its conclusions. 